\title{
Classifying superconductivity in ThH-ThD superhydrides
}

\author{
E. F. Talantsev ${ }^{1,2}$ \\ ${ }^{1}$ M.N. Mikheev Institute of Metal Physics, Ural Branch, Russian Academy of Sciences, \\ 18, S. Kovalevskoy St., Ekaterinburg, 620108, Russia \\ ${ }^{2}$ NANOTECH Centre, Ural Federal University, 19 Mira St., Ekaterinburg, 620002, \\ Russia
}

E-mail: evgeny.talantsev@imp.uran.ru

\begin{abstract}
Satterthwaite and Toepke (1970 Phys. Rev. Lett. 25 741) discovered that $\mathrm{Th}_{4} \mathrm{H}_{15}-\mathrm{Th}_{4} \mathrm{D}_{15}$ superhydrides exhibit superconductivity and have no isotope effect. The latter is fundamental contradiction with the concept of electron-phonon mediated superconductivity of BardeenCooper-Schrieffer (BCS) theory. Soon after this work, Stritzker and Buckel (1972 Zeitschrift für Physik A Hadrons and nuclei 257 1-8) reported that superconductors in $\mathrm{PdH}_{\mathrm{x}}-\mathrm{PdD}_{\mathrm{x}}$ system exhibit reverse isotope effect. Yussouff et al (1995 Solid State Communications 94 549) extended this finding on $\mathrm{PdH}_{\mathrm{x}}-\mathrm{PdD}_{\mathrm{x}}-\mathrm{PdT}_{\mathrm{x}}$ system. Recent interest to hydrogen- and deuterium-rich superconductors is based on the discovery of near-room-temperature superconductivity in highly-compressed $\mathrm{H}_{3} \mathrm{~S}$ (Drozdov et al. 2015 Nature 525 73) and $\mathrm{LaH}_{10}$ (Somayazulu et al 2019 Phys. Rev. Lett. 122 027001). To date, there is no clarity about isotope effect in $\mathrm{H}_{3} \mathrm{~S}-\mathrm{D}_{3} \mathrm{~S}$ system, because thorough examination of available experimental data reported by Drozdov et al (2015 Nature $\mathbf{5 2 5} 73$ ) shows that $\mathrm{H}_{3} \mathrm{~S}_{-} \mathrm{D}_{3} \mathrm{~S}$ system perhaps has reverse isotope effect. In attempt to reaffirm/disprove our primary idea that the mechanism for near-room-temperature superconductivity in hydrogen-rich superconductors is not BCS electron-phonon interaction, we analyse the upper critical field data, $B_{\mathrm{c} 2}(T)$, in $\mathrm{Th}_{4} \mathrm{H}_{15}-\mathrm{Th}_{4} \mathrm{D}_{15}$ phases (Satterthwaite and Toepke 1970 Phys. Rev. Lett. 25 741) and two recently discovered high-pressure hydrogen-rich phases of $\mathrm{ThH}_{9}$ and $\mathrm{ThH}_{10}$ (Semenok et al 2019
\end{abstract}




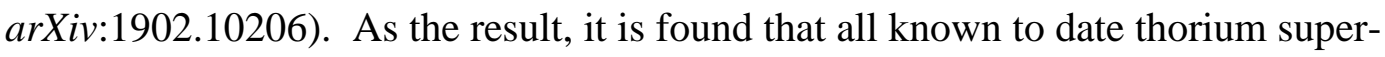
hydrides/deuterides are unconventional superconductors which have $T_{\mathrm{c}} / T_{\mathrm{F}}$ ratios within a range of $0.008<T_{\mathrm{c}} / T_{\mathrm{F}}<0.120$, where $T_{\mathrm{c}}$ is the superconducting transition temperature and $T_{\mathrm{F}}$ is the Fermi temperature.

\section{Introduction}

The isotope effect in Bardeen-Cooper-Schrieffer (BCS) theory of superconductivity expressed in the form:

$$
T_{c} \cdot M^{\alpha}=\text { const. }
$$

where $M$ is isotope mass, and $\alpha \approx 1 / 2$ (for weak-coupling limit of BCS theory [1]), is central indispensable feature of electron-phonon mediated superconductivity [1]. This effect was observed in several elemental superconductors, but not in all of them [2]. And, for instance, Geballe et al [3] were first who found the absence of the isotope effect in ruthenium (more details can be found elsewhere [2-4]). Later, Satterthwaite and Toepke [5] reported the absence of the isotope effect in $\mathrm{Th}_{4} \mathrm{H}_{15}-\mathrm{Th}_{4} \mathrm{D}_{15}$ super-hydride/deuteride phases. Soon after [5], Stritzker and Buckel [6] experimentally found that the isotope effect in the palladiumhydrogen-deuterium $\left(\mathrm{PdH}_{\mathrm{x}}-\mathrm{PdD}_{\mathrm{x}}\right)$ system has opposite sign (so called, reverse isotope effect). Yussouff et al [7] extended this discovery on palladium-hydrogen-deuterium-tritium system $\left(\mathrm{PdH}_{\mathrm{x}}-\mathrm{PdD}_{\mathrm{x}}-\mathrm{PdT}_{\mathrm{x}}\right)$. This reverse isotope effect in $\mathrm{PdH}_{\mathrm{x}}-\mathrm{PdD}_{\mathrm{x}}-\mathrm{PdT}_{\mathrm{x}}$ system is still under wide discussion $[8,9]$. In regard of considered in this paper ThH-ThD system, detailed studied by Caton and Satterthwaite [10] showed that superconductors in thorium-hydrogen-deuterium (ThH-ThD) system have reverse isotope effect, and Dietrich et al [11] showed that transition temperature, $T_{c}$, of the $\mathrm{Th}_{4} \mathrm{H}_{15}$ phase has linear positive ramp of $0.42 \mathrm{~K} / \mathrm{GPa}$ versus applied external pressure. 
Recent interest to the isotope effect in superconducting compounds based on isotopes of hydrogen is based on experimental discovery of near-room-temperature superconductivity in $\mathrm{H}_{3} \mathrm{~S}-\mathrm{D}_{3} \mathrm{~S}$ [12] and $\mathrm{LaH}_{10}$ [13]. It should be stressed that to date there is no clarity on the isotope effect in $\mathrm{H}_{3} \mathrm{~S}-\mathrm{D}_{3} \mathrm{~S}$ system. Truly, despite a fact that Drozdov et al [12] in their Fig. 2(b) showed $R(T)$ curves for $\mathrm{H}_{3} \mathrm{~S}$ and $\mathrm{D}_{3} \mathrm{~S}$ which demonstrate much lower $T_{\mathrm{c}}$ for $\mathrm{D}_{3} \mathrm{~S}$ in compassion with $\mathrm{H}_{3} \mathrm{~S}$, through examination of this plot shows that $R(T)$ curves for these two compounds were measured at crucially different pressures of $P=141 \mathrm{GPa}$ and $P=155 \mathrm{GPa}$. To make direct comparison, $R(T)$ curves for both compounds should be measured at the same pressure. Thoroughly examination of available $R(T)$ curves measured at the same pressure for $\mathrm{H}_{3} \mathrm{~S}$ and $\mathrm{D}_{3} \mathrm{~S}$ reveals that $\mathrm{H}_{3} \mathrm{~S}-\mathrm{D}_{3} \mathrm{~S}$ system has remarkably large reverse isotope effect.

In Fig. 1 we show in $R(T)$ curves for $\mathrm{H}_{3} \mathrm{~S}$ and $\mathrm{D}_{3} \mathrm{~S}$ samples measured at $P=155 \mathrm{GPa}$. Two $R(T)$ curves for $\mathrm{H}_{3} \mathrm{~S}$ were taken from Fig. 1(a) and Fig. 3(a) of Drozdov et al [12]. And the $R(T)$ curve for $\mathrm{D}_{3} \mathrm{~S}$ was taken from Fig. 2(b). There is clear experimental result that $\mathrm{H}_{3} \mathrm{~S}-$ $\mathrm{D}_{3} \mathrm{~S}$ system has reverse isotope effect. Due to $R(T)$ curves recorded at the same pressure for $\mathrm{H}_{3} \mathrm{~S}$ and $\mathrm{D}_{3} \mathrm{~S}$ (which we demonstrate in Fig. 1) are only ones available to date, the only conclusion which can be made is that widely accepted point of view that high-temperature superconductivity in $\mathrm{H}_{3} \mathrm{~S}$ and $\mathrm{D}_{3} \mathrm{~S}$ is electron-phonon interaction [14] does not have support in isotope effect experiments.

This finding is additional support to our previous proposal that hydrogen-rich compounds (for instance, $\mathrm{PdH}_{x}, \mathrm{H}_{3} \mathrm{~S}, \mathrm{LaH}_{10}$ ) are unconventional superconductors $[15,16]$ and the superconductivity in these compounds is not related to electron-phonon interaction.

We should note that in our consideration we do not include:

1. Highly compressed silane $\mathrm{SiH}_{4}$ (this was the first discovered by Eremets group highly-compressed hydrogen-rich superconductor with $T_{c}=17 \mathrm{~K}$ (observed at pressure of $P=96-120 \mathrm{GPa})[17]$. 
2. Covalent hydride phosphine, $\mathrm{PH}_{3}$, is another hydrogen-rich superconductor in which superconductivity with $T_{c} \simeq 100 \mathrm{~K}$ was discovered at $P \gtrsim 200 \mathrm{GPa}$ [18].

3. $\operatorname{PtH}_{x}(x \cong 1)$ which was recently reported to be superconducting at $P=30 \mathrm{GPa}$ [19].

4. $\mathrm{NbTiH}_{x}[20]$ is another hydrogen-rich superconductor which can be potentially considered.

The reason why these interesting materials are not under our consideration, is that for all of these compounds, fundamental experimental data beyond $T_{\mathrm{c}}$ are unknown, and, thus, we were not able to analyse these materials in our consideration herein.

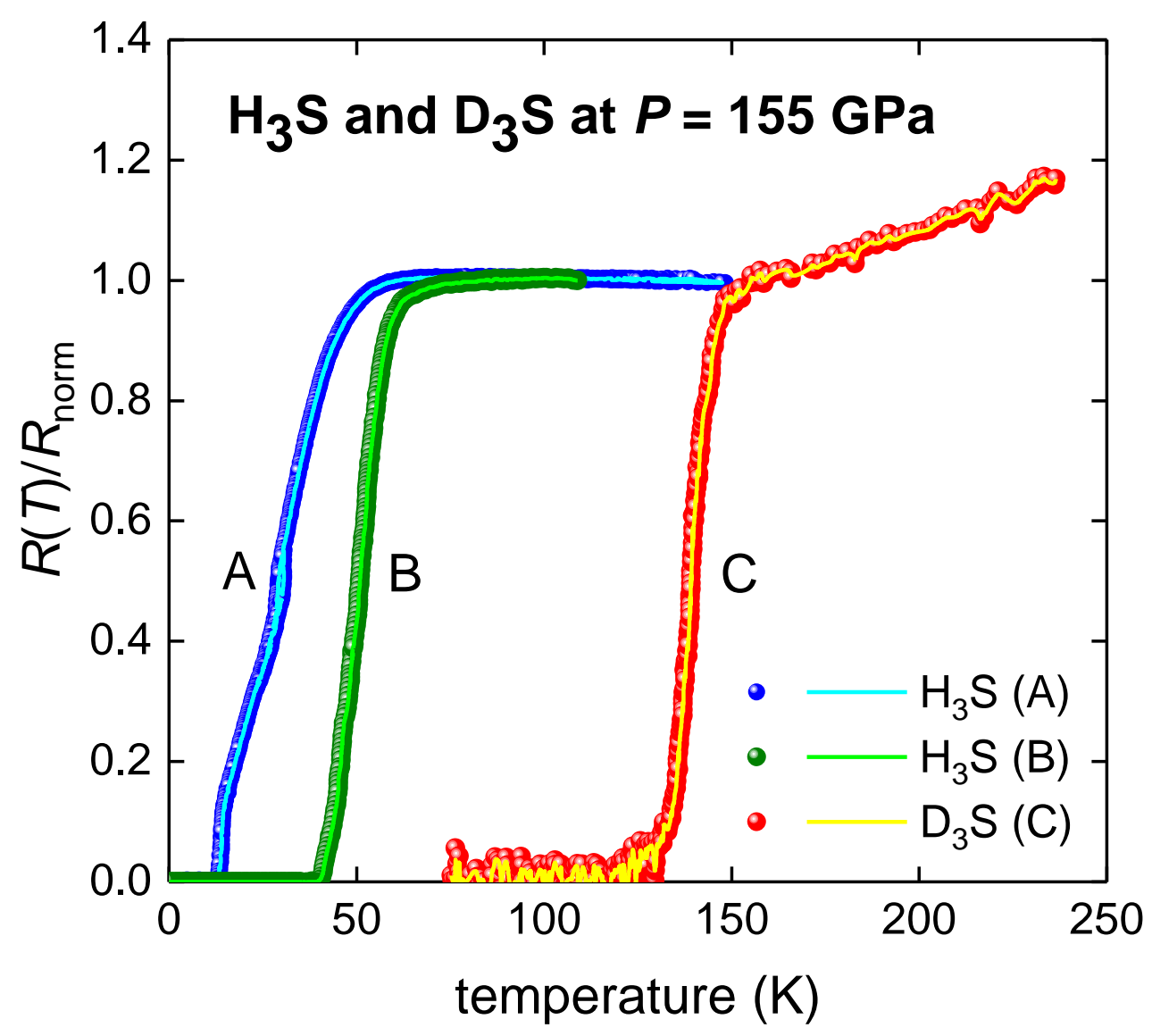

Figure 1. $R(T)$ curves for $\mathrm{H}_{3} \mathrm{~S}(\mathrm{~A}, \mathrm{~B})$ and $\mathrm{D}_{3} \mathrm{~S}(\mathrm{C})$ measured at $P=155 \mathrm{GPa}$. Data for $\mathrm{A}$ was taken from Fig. 3(a) [12]; data for B was taken from Fig. 1(a) [12]; data for $\mathrm{C}$ was taken from Fig. 2(b) [12].

In result, in this paper we show that all discovered to date hydrogen-rich superconductors for which experimental data beyond $T_{\mathrm{c}}$ are available, i.e. $\mathrm{PdH}_{\mathrm{x}}, \mathrm{Th}_{4} \mathrm{H}_{15}, \mathrm{Th}_{4} \mathrm{D}_{15}, \mathrm{ThH}_{9}, \mathrm{ThH}_{10}$, 
$\mathrm{H}_{3} \mathrm{~S}$ and $\mathrm{LaH}_{10}$ are unconventional superconductors which lie in the same band in the Uemura plot as all other unconventional superconductors (particularly heavy fermions, fullerenes, pnictides, and cuprates).

It should be stressed, that in some scenarios, $\mathrm{Th}_{4} \mathrm{H}_{15}$ and $\mathrm{Th}_{4} \mathrm{D}_{15}$ are located in closed proximity to Bose-Einstein condensate (BEC) line in the Uemura plot.

\section{The upper critical field models}

Ground state upper critical field, $B_{\mathrm{c} 2}(0)$, in the Ginzburg-Landau theory [21] is given by:

$$
B_{c 2}\left(\frac{T}{T_{c}}=0\right)=\frac{\phi_{0}}{2 \cdot \pi \cdot \xi^{2}(0)}
$$

where $\phi_{0}=2.068 \cdot 10^{-15} \mathrm{~Wb}$ is magnetic flux quantum, and $\xi(0)$ is the ground state coherence length. For real world experiments, when, as a rule, only a part of full $B_{\mathrm{c} 2}(T)$ temperature dependence can be measured, there are several models were proposed to deduce extrapolated values for $\xi(0)$ from raw $B_{\mathrm{c} 2}(T)$ data measured at high reduced temperatures.

One of the model, which was proposed by Werthamer, Helfand, and Hohenberg [22,23], is extrapolative expression:

$$
B_{c 2}(0)=\frac{\phi_{0}}{2 \cdot \pi \cdot \xi^{2}(0)}=-0.693 \cdot T_{C} \cdot\left(\frac{d B_{c 2}(T)}{d T}\right)_{T \sim T_{C}}
$$

In this paper, we will designate Eq. 2 as WHH model.

Another model, which is based on WHH primary idea [22,23], but one accurately generates full $B_{\mathrm{c} 2}(T)$ extrapolative curve from experimental data measured at high reduced temperatures, $T / T_{\mathrm{c}}$, was proposed by Baumgartner et al [24]:

$$
B_{c 2}(T)=\frac{\phi_{0}}{2 \cdot \pi \cdot \xi^{2}(0)} \cdot\left(\frac{\left(1-\frac{T}{T_{C}}\right)-0.153 \cdot\left(1-\frac{T}{T_{C}}\right)^{2}-0.152 \cdot\left(1-\frac{T}{T_{C}}\right)^{4}}{0.693}\right)
$$

We will designate this model as B-WHH model.

Gor'kov [25] proposed $B_{\mathrm{c} 2}(T)$ model which we used in our previous papers $[15,16]$ : 


$$
B_{c 2}(T)=\frac{\phi_{0}}{2 \cdot \pi \cdot \xi^{2}(0)} \cdot\left(\frac{1.77-0.43 \cdot\left(\frac{T}{T_{C}}\right)^{2}+0.07 \cdot\left(\frac{T}{T_{C}}\right)^{4}}{1.77}\right) \cdot\left[1-\left(\frac{T}{T_{C}}\right)^{2}\right]
$$

We will designate this model as G-model.

Jones et al [26], proposed so called Jones-Hulm-Chandrasekhar (JHC) model:

$$
B_{c 2}(T)=\frac{\phi_{0}}{2 \cdot \pi \cdot \xi^{2}(0)} \cdot\left(\frac{1-\left(\frac{T}{T_{c}}\right)^{2}}{1+\left(\frac{T}{T_{c}}\right)^{2}}\right)
$$

\section{Th4 $4 H_{15}-\mathrm{Th}_{4} \mathrm{D}_{15}$ superconductors in Uemura plot}

We start our consideration with the first discovered superhydride/superdeuteride superconductors, i.e., $\mathrm{Th}_{4} \mathrm{H}_{15}$ and $\mathrm{Th}_{4} \mathrm{D}_{15}$ [5]. From the author's knowledge, available to date experimental data for the upper critical field, $B_{\mathrm{c} 2}(T)$, for $\mathrm{Th}_{4} \mathrm{H}_{15}$ and $\mathrm{Th}_{4} \mathrm{D}_{15}$ are limited by values reported by Satterthwaite and Toepke [5], who measured that both $\mathrm{Th}_{4} \mathrm{H}_{15}$ and $\mathrm{Th}_{4} \mathrm{D}_{15}$ compounds have ground state upper critical field:

$$
B_{c 2}(T \sim 0)=2.5-3.0 T \text {. }
$$

From these values, the ground state coherence length, $\xi(0)$, for $\mathrm{Th}_{4} \mathrm{H}_{15}$ and $\mathrm{Th}_{4} \mathrm{D}_{15}$ phases, can be derived as following:

$$
\xi(0)=11.0 \pm 0.5 \mathrm{~nm}
$$

Miller et al. [27] for both phases reported the BCS ratio within a range:

$$
\alpha=\frac{2 \cdot \Delta(0)}{k_{B} \cdot T_{C}}=3.42-3.47
$$

By utilizing superconducting transition temperature for $\mathrm{Th}_{4} \mathrm{H}_{15}$ and $\mathrm{Th}_{4} \mathrm{D}_{15}$ phases [5]:

$$
T_{c}=8.20 \pm 0.15 \mathrm{~K}
$$

one can deduce ground state superconducting energy gap:

$$
\Delta(0)=1.22 \pm 0.03 \mathrm{meV}
$$

and by using well-known BCS expression [1]:

$$
\xi(0)=\frac{\hbar \cdot v_{F}}{\pi \cdot \Delta(0)}
$$


where $\hbar=h / 2 \pi$ is reduced Planck constant, one can calculate the Fermi velocity, $v_{\mathrm{F}}$, in $\mathrm{Th}_{4} \mathrm{H}_{15}$ and $\mathrm{Th}_{4} \mathrm{D}_{15}$ phases:

$$
v_{F}=\pi \cdot \frac{\xi(0) \cdot \Delta(0)}{\hbar}=(6.4 \pm 0.2) \cdot 10^{4} \mathrm{~m} / \mathrm{s}
$$

To place $\mathrm{Th}_{4} \mathrm{H}_{15}$ and $\mathrm{Th}_{4} \mathrm{D}_{15}$ phases in the Uemura plot $[28,29]$, we need to make assumption about the effective charge carrier mass, $m_{e f f}^{*}$, to calculate the Fermi temperature, $T_{\mathrm{F}}:$

$$
T_{F}=\frac{\varepsilon_{F}}{k_{B}}=\frac{m_{e f f}^{*} \cdot v_{F}^{2}}{2 \cdot k_{B}}
$$

Due to there is no any available experimental $m_{\text {eff }}^{*}$ values for $\mathrm{Th}_{4} \mathrm{H}_{15}$ and $\mathrm{Th}_{4} \mathrm{D}_{15}$ phases, we can use for the lower bound of $m_{e f f}^{*}$ the value for another ambient pressure hydrogen-rich superconductor, $\mathrm{PdH}_{\mathrm{x}}[30]$ :

$$
m_{e f f}^{*}=0.49 \cdot m_{e}
$$

and one calculates the Fermi temperature, $T_{\mathrm{F}}$ :

$$
T_{F}=\frac{\varepsilon_{F}}{k_{B}}=\frac{m_{e f f}^{*} \cdot v_{F}^{2}}{2 \cdot k_{B}}=67 \pm 4 K
$$

and the ratio:

$$
\frac{T_{C}}{T_{F}}=0.12 \pm 0.01
$$

For the upper bond of $m_{e f f}^{*}$ value we used the highest value reported for highly compressed hydrides, $m_{e f f}^{*}=3.0 \cdot m_{e}[31]$, and the lower bound for the $T_{\mathrm{c}} / T_{\mathrm{F}}$ value can be calculated as:

$$
\frac{T_{C}}{T_{F}}=0.020 \pm 0.002
$$

Thus, performed analysis shows (Fig. 2) that in the scenario of $m_{e f f}^{*}=0.49 \cdot m_{e}, \mathrm{Th}_{4} \mathrm{H}_{15}$ and $T h_{4} \mathrm{D}_{15}$ phases are located in the Uemura plot in the closed proximity to Bose-Einstein condensate (BEC) superfluid line together with ${ }^{4} \mathrm{He}$ and ${ }^{40} \mathrm{~K}$, and thus these two phases cannot be obey BCS theory. For the scenario of $m_{e f f}^{*}=3.0 \cdot m_{e}, \mathrm{Th}_{4} \mathrm{H}_{15}$ and $\mathrm{Th}_{4} \mathrm{D}_{15}$ phases are still within unconventional superconductor band in the Uemura plot (Fig. 2), where all 
unconventional superconductors (i.e. heavy fermions, fullerens, pnictides and cuprates) are located.

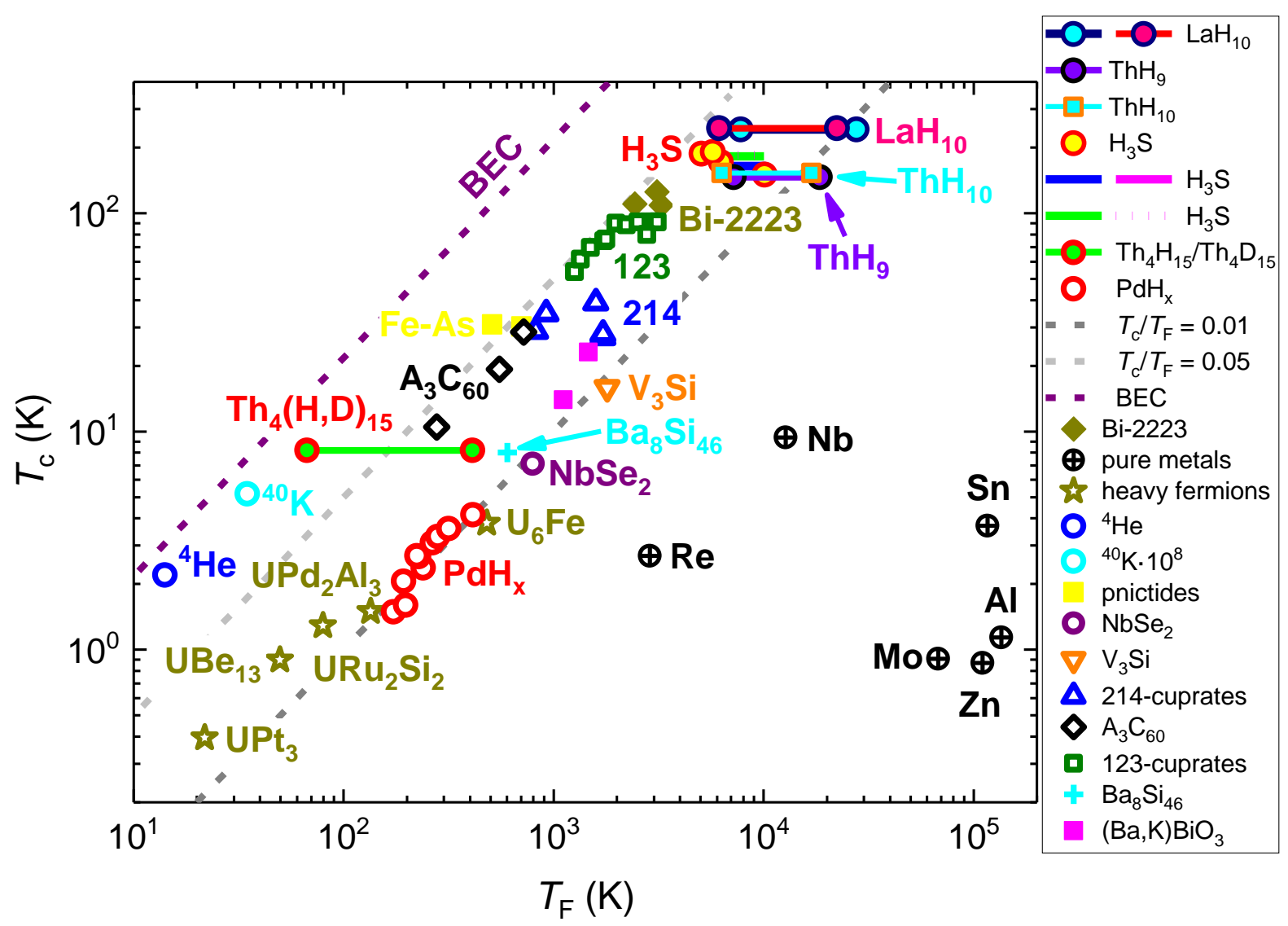

Figure 2. A plot of $T_{\mathrm{c}}$ versus $T_{\mathrm{F}}$ obtained for most representative superconducting families including $\mathrm{PdH}_{\mathrm{x}}, \mathrm{Th}_{4} \mathrm{H}_{15} / \mathrm{Th}_{4} \mathrm{D}_{15}, \mathrm{ThH}_{9}, \mathrm{ThH}_{10}, \mathrm{H}_{3} \mathrm{~S}$, and $\mathrm{LaH}_{10}$. Data was taken from Uemura [29], Ye et al. [32], Qian et al. [33], Hashimoto et al. [34] and Refs. 15,16.

\section{ThH9 $(P=170 \mathrm{GPa})$ in Uemura plot}

Semenok et al [35] reported on the discovery of high-temperature superconducting phase of $\mathrm{ThH}_{9}$ at $P=170 \mathrm{GPa}$ which exhibits $P 6_{3} / m m c$ crystallographic symmetry and superconducting transition temperature of $T_{\mathrm{c}}=146 \mathrm{~K}$. Semenok et al [35] also performed first principles calculations and deduced the effective mass in this superconductor:

$$
m_{e f f}^{*}=2.73 \cdot m_{e}
$$

which is remarkably close to the effective mass of $m_{e f f}^{*}=2.76 \cdot m_{e}$ in compressed $\mathrm{H}_{3} \mathrm{~S}$ [36]. 
Semenok et al [35] also proposed that $\mathrm{ThH}_{9}$ has $\mathrm{BCS}$ ratio:

$$
\alpha=\frac{2 \cdot \Delta(0)}{k_{B} \cdot T_{C}}=4.74-4.89
$$

It should be noted, that as we already mentioned in our previous papers $[15,16,37,38]$, that first principles calculations [31,35,36,39-41] always provide $\alpha$-values near 5, which is the range of very strong-coupling limit for $s$-wave symmetry (it should be noted that other superconducting gap symmetries have weak-coupling limits of $\alpha \sim 5$ [42-44]).

However, it is needed to be mentioned, that first there are several new alternative approaches were developed to explain near-room-temperature superconductivity in compressed hydrides (we can mention, Hirsch and Marsiglio [45], Souza and Marsiglio [46], Harshman and Fiory [47], Kaplan and Imry [48]). For instance, Kaplan and Imry [48] showed that for the case of highly compressed $\mathrm{H}_{3} \mathrm{~S}$ their model gives $\alpha$ within weak-coupling BCS limit:

$$
\alpha=\frac{2 \cdot \Delta(0)}{k_{B} \cdot T_{C}}=3.53
$$

This $\alpha$ value is in a good agreement with ones deduced from experimental $B_{\mathrm{c} 2}(T)$ [15] and the self-field critical current density, $J_{\mathrm{c}}(\mathrm{sf}, T)$, data [,37,49]. Assuming that all hydrogen-rich superconductors have similar origin for the superconductivity, the value of $a=3.53$ was used in our calculations as the lowest boundary value for $\alpha$.

Semenok et al [35] measured $B_{\mathrm{c} 2}(T)$ data for $\mathrm{ThH}_{9}$ phase at $P=170 \mathrm{GPa}$, which we fit to Eqs. 2-5 in Fig. 3. It can be seen that deduced $T_{\mathrm{c}} / T_{\mathrm{F}}$ ratios are within usual range of unconventional superconductors band (Fig. 2). 


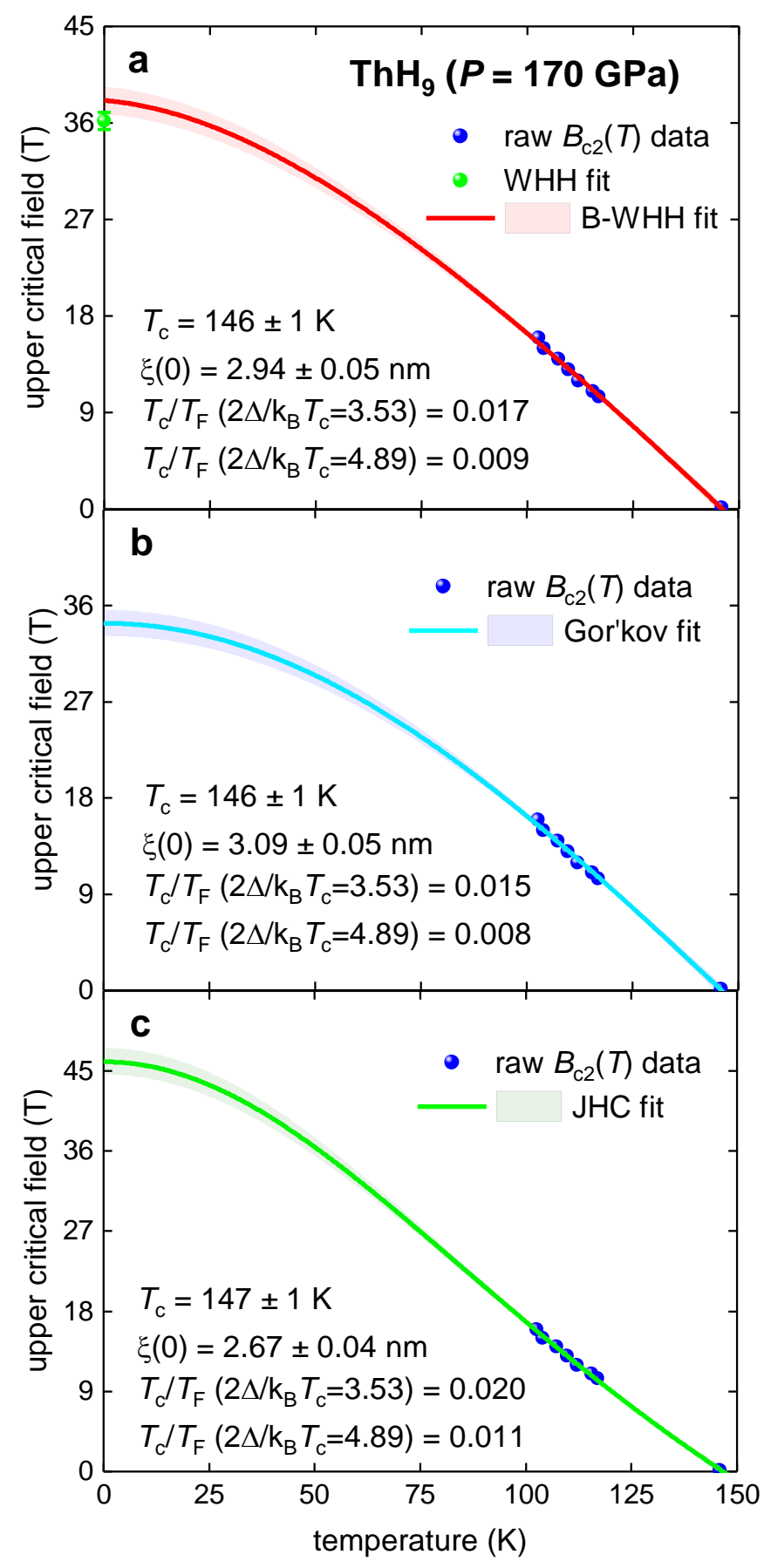

Figure 3. Superconducting upper critical field, $B_{\mathrm{c} 2}(T)$, data and fits to four different model (Eqs. 2-5) for $\mathrm{ThH}_{9}$ superhydride compressed at pressure $P=170 \mathrm{GPa}$ (raw data are from Ref. 35). (a) fit to WHH and B-WHH models, for latter the fit quality is $R=0.998$; (b) fit to Gor'kov model, $R=0.998$; (c) fit to JHC model, $R=0.9988$. 95\% confidence bars are shown.

\section{ThH10 $(P=174$ GPa $)$ in Uemura plot}

Semenok et al [35] also reported on the discovery of another high-temperature superconducting phase of $\mathrm{ThH}_{10}$ at $P=174 \mathrm{GPa}$, which exhibits $F m \overline{3} m$ crystallographic 
symmetry and superconducting transition temperature of $T_{\mathrm{c}}=159 \mathrm{~K}$. In Fig. 4 we show raw upper critical field, $B_{\mathrm{c} 2}(T)$, data for this phase [35] and data fit to Eqs. 2-5.

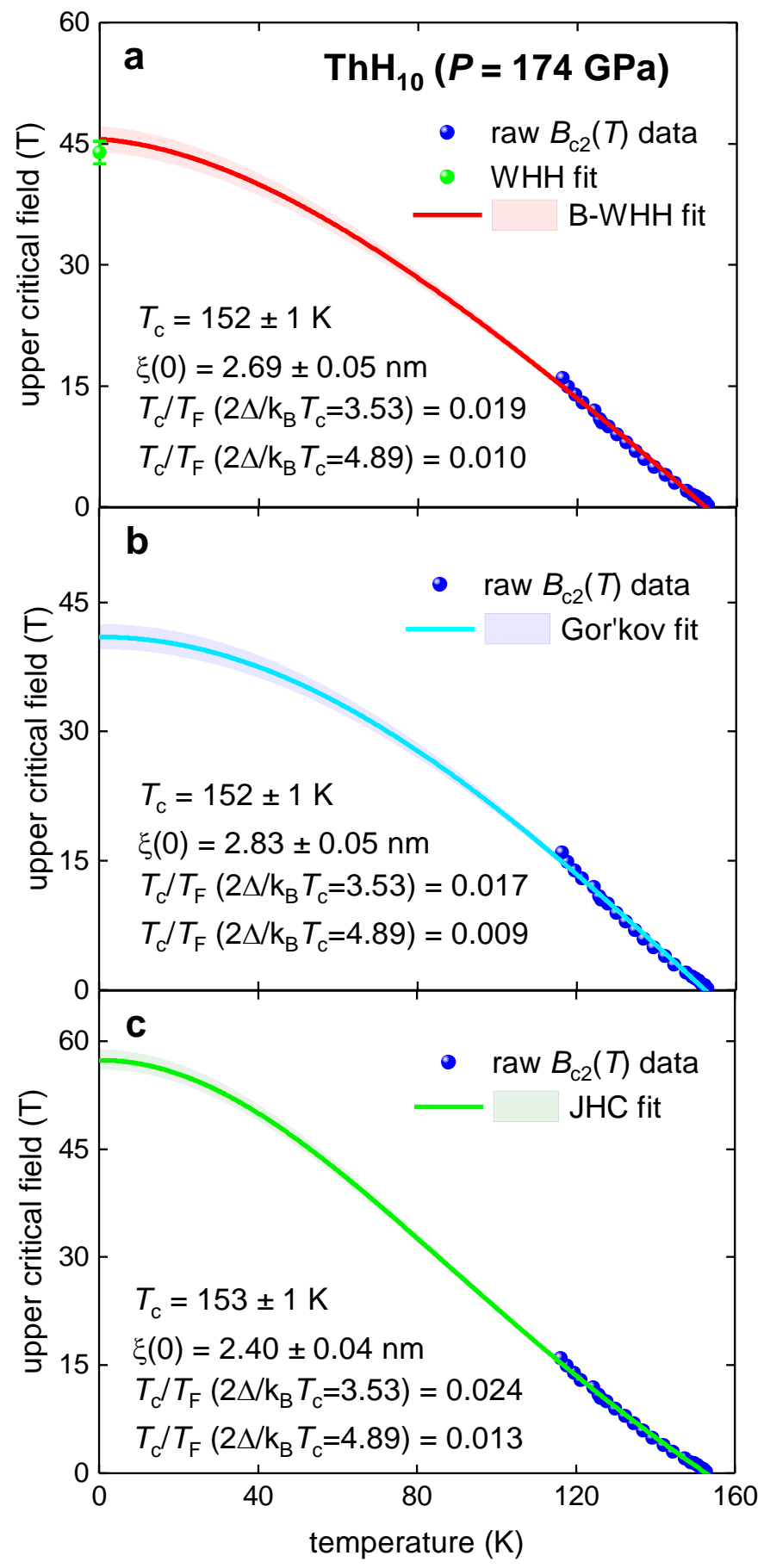

Figure 4. Superconducting upper critical field, $B_{\mathrm{c} 2}(T)$, data and fits to four different model (Eqs. 2-5) for $\mathrm{ThH}_{10}$ superhydride compressed at pressure $P=174 \mathrm{GPa}$ (raw data are from Ref. 35). (a) fit to WHH and B-WHH models, for latter the fit quality is $R=0.992$; (b) fit to Gor'kov model, $R=0.992$; (c) fit to JHC model, $R=0.997$. 95\% confidence bars are shown. 
As expected, in the Uemura plot highly-compressed $\mathrm{ThH}_{10}$ superconductor is located within unconventional superconductors band (Fig. 2).

\section{Conclusions}

In this paper we analyse experimental $B_{\mathrm{c} 2}(T)$ data for several thorium based superhydrides and $\mathrm{Th}_{4} \mathrm{D}_{15}$ superdeuteride and come to conclusion that all discovered to date thorium hydrogen- and deuterium-rich superconductors for which fundamental superconducting parameters beyond $T_{\mathrm{c}}$ have measured, i.e., $\mathrm{Th}_{4} \mathrm{H}_{15}, \mathrm{Th}_{4} \mathrm{D}_{15}, \mathrm{ThH}_{9}$ and $\mathrm{ThH}_{10}$, are unconventional superconductors.

In addition, we stress that the isotope effect in $\mathrm{H}_{3} \mathrm{~S}-\mathrm{D}_{3} \mathrm{~S}$ system should be further studied, because available to date experimental data are not sufficient to make solid conclusion.

\section{Acknowledgement}

Author thanks financial support provided by the state assignment of Minobrnauki of Russia (theme "Pressure" No. AAAA-A18-118020190104-3) and by Act 211 Government of the Russian Federation, contract No. 02.A03.21.0006.

\section{References}

[1] Bardeen J, Cooper L N, Schrieffer J R 1957 Theory of superconductivity Phys. Rev. 108, 1175-1204

[2] Poole P P, Farach H A, Creswick R J, Prozorov R 2007 Superconductivity (2-nd Edition, London, UK)

[3] Geballe T H, Matthias B T, Hull G W, Jr, and Corenzwit E 1961 Absence of an isotope effect in superconducting ruthenium Phys. Rev. Lett. 6 275-277

[4] Hirsch J E 2019 Superconducting materials: the whole story arXiv:1908.04419

[5] Satterthwaite C B and Toepke I L 1970 Superconductivity of hydrides and deuterides of thorium Phys. Rev. Lett. 25 741-743

[6] Stritzker B and Buckel W 1972 Superconductivity in the palladium-hydrogen and the palladium-deuterium systems Zeitschrift für Physik A Hadrons and nuclei 257 1-8

[7] Yussouff M, Rao B K and Jena P 1995 Reverse isotope effect on the superconductivity of PdH, PdD, and PdT Solid State Communications 94 549-553 
[8] Villa-Cortés S and Baquero R 2018 On the calculation of the inverse isotope effect in PdH(D): A Migdal-Eliashberg theory approach Journal of Physics and Chemistry of Solids 119 80-84

[9] Ostanin S, Borisov V, Fedorov D V, Salamatov E I, Ernst A and Mertig I 2019 Role of tetrahedrally coordinated dopants in palladium hydrides on their superconductivity and inverse isotope effect Journal of Physics: Condensed Matter 31075703

[10] Caton R and Satterthwaite C B 1977 Preparation and characterization of massive $\mathrm{Th}_{4} \mathrm{H}_{15}$ and $\mathrm{Th}_{4} \mathrm{D}_{15}$ Journal of the Less Common Metals 52307

[11] Dietrich M, Gey W, Rietschel H, Satterthwaite C B 1974 Pressure dependence of the superconducting transition temperature of $\mathrm{Th}_{4} \mathrm{H}_{15}$ Solid State Communications 15 941-943.

[12] Drozdov A P, Eremets M I, Troyan I A, Ksenofontov V, Shylin S I 2015 Conventional superconductivity at 203 kelvin at high pressures in the sulfur hydride system Nature $\mathbf{5 2 5} 73$ 76

[13] Somayazulu M, Ahart M, Mishra A K, Geballe Z M, Baldini M, Meng Y, Struzhkin V V and R. J. Hemley R J 2019 Evidence for superconductivity above $260 \mathrm{~K}$ in lanthanum superhydride at megabar pressures Phys. Rev. Lett. 122027001

[14] Mazin I I 2015 Extraordinarily conventional Nature 525 40-41

[15] Talantsev E F 2019 Classifying superconductivity in compressed $\mathrm{H}_{3} \mathrm{~S}$ Modern Physics Letters B 331950195

[16] Talantsev E F 2019 Classifying hydrogen-rich superconductors Materials Research Express 6106002

[17] Eremets M I, Trojan I A, Medvedev S A, Tse J S, Yao Y 2008 Superconductivity in hydrogen dominant materials: Silane Science 319 1506-1509

[18] Drozdov A P, Eremets M I, Troyan I A 2015 Superconductivity above $100 \mathrm{~K}$ in $\mathrm{PH}_{3}$ at high pressures arXiv:1508.06224

[19] Matsuoka T, et al. 2019 Superconductivity of platinum hydride Phys Rev B 99144511

[20] Antonov V E, Belash I T, Zakharov M S, Orlov V A, Rashupkin V I 1986 Int. J.

Hydrogen Energy 11475.

[21] Ginzburg V L and Landau L D 1950 On the theory of superconductivity Zh. Eksp. Teor. Fiz. 20 1064-1082

[22] Helfand E and Werthamer N R 1966 Temperature and purity dependence of the superconducting critical field, $H_{\mathrm{c} 2}$. II. Phys. Rev. 147 288-294

[23] Werthamer N R, Helfand E and Hohenberg P C 1966 Temperature and purity dependence of the superconducting critical field, $H_{\mathrm{c} 2}$. III. Electron spin and spin-orbit effects Phys. Rev. 147 295-302

[24] Baumgartner T, Eisterer M, Weber H W, Fluekiger R, Scheuerlein C, Bottura L 2014

Effects of neutron irradiation on pinning force scaling in state-of-the-art $\mathrm{Nb}_{3} \mathrm{Sn}$ wires

Supercond. Sci. Technol. 27015005

[25] Gor'kov L P 1960 The critical supercooling field in superconductivity theory Soviet Physics JETP 10 593-599

[26] Jones C K, Hulm J K, Chandrasekhar B S 1964 Upper critical field of solid solution alloys of the transition elements Rev. Mod. Phys. 36 74-76

[27] Miller J F, Caton R H, Satterthwaite C B 1976 Low-temperature heat capacity of normal and superconducting thorium hydride and thorium deuteride. Phys. Rev. B 142795

[28] Uemura Y J, et al 1989 Universal correlations between $T_{\mathrm{c}}$ and $\frac{n_{s}}{m^{*}}$ (carrier density over effective mass) in high- $T_{\mathrm{c}}$ cuprate Phys. Rev. Lett. 62 2317-2320

[29] Uemura Y J 2004 Condensation, excitation, pairing, and superfluid density in high- $T_{\mathrm{c}}$ superconductors: the magnetic resonance mode as a roton analogue and a possible spinmediated pairing J. Phys.: Condens. Matter 16 S4515-S4540 
[30] Bambakidis G, Smith R J, and Otterson D A 1968 Electrical resistivity as a function of deuterium concentration in palladium NASA Report Number TN D-4970

[31] Kostrzewa M, Szczesniak K M, Durajski A P and Szczesniak R 2019 From $\mathrm{LaH}_{10}$ to room-temperature superconductors arXiv:1905.12308

[32] Ye J T, et al. 2012 Superconducting dome in a gate-tuned band insulator Science 338 1193

[33] Qian T, et al. 2011 Absence of a holelike Fermi surface for the iron-based $\mathrm{K}_{0.8} \mathrm{Fe}_{1.7} \mathrm{Se}_{2}$ superconductor revealed by angle-resolved photoemission spectroscopy Phys. Rev. Lett. 106 187001

[34] Hashimoto K, Cho K, Shibauchi T, Kasahara S, Mizukami Y, Katsumata R, Tsuruhara Y, Terashima T, Ikeda H, Tanatar M A, Kitano H, Salovich N, Giannetta R W, Walmsley P, Carrington A, Prozorov R, Matsuda Y 2012 A sharp peak of the zero-temperature penetration depth at optimal composition in $\mathrm{BaFe}_{2}\left(\mathrm{As}_{1-\mathrm{x}} \mathrm{P}_{\mathrm{x}}\right)_{2}$ Science 336 1554-1557

[35] Semenok D V, Kvashnin A G, Ivanova A G, Svitlyk V, Fominski V Yu, Sadakov A V, Sobolevskiy O A, Pudalov V M, Troyan I A and Oganov A R 2019 Superconductivity at 161 $\mathrm{K}$ in thorium hydride $\mathrm{ThH}_{10}$ : Synthesis and properties arXiv:1902.10206

[36] Durajski A P 2016 Quantitative analysis of nonadiabatic effects in dense $\mathrm{H}_{3} \mathrm{~S}$ and $\mathrm{PH}_{3}$ superconductors Scientific Reports 638570

[37] Talantsev E F, Crump W P, Storey J G, Tallon J L 2017 London penetration depth and thermal fluctuations in the sulphur hydride $203 \mathrm{~K}$ superconductor Annalen der Physics $\mathbf{5 2 9}$ 1600390

[38] Tallon J L and Talantsev E F 2018 Compressed $\mathrm{H}_{3}$ S, superfluid density and the quest for room-temperature superconductivity Journal of Superconductivity and Novel Magnetism 31 619-624

[39] Kostrzewa M, Szczesniak K M, Durajski A P, Szczesniak R 2019 From $\mathrm{LaH}_{10}$ to roomtemperature superconductors arXiv:1905.12308

[40] Nicol E J and Carbotte J P 2015 Comparison of pressurized sulfur hydride with conventional superconductors Phys. Rev. B 91 220507(R)

[41] Troyan I A, Semenok D V, Kvashnin A G, Ivanova A G, Prakapenka V B, Greenberg E, Gavriliuk A G, Lyubutin I S, Struzhkin V V and Oganov A R 2019 Synthesis and superconductivity of yttrium hexahydride $\mathrm{Im} \overline{3 m}-\mathrm{YH}_{6}$ arXiv:1908.01534

[42] Gross F, Chandrasekhar B S, Einzel D, Andres K, Hirschfeld P J, Ott H R, Beuers J,

Fisk Z, Smith J L 1986 Anomalous temperature dependence of the magnetic field penetration depth in superconducting $\mathrm{UBe}_{3} Z$ Z. Phys. B - Condensed Matter 64 175-188

[43] Gross-Alltag F, Chandrasekhar B S, Einzel D, Hirschfeld P J, Andres K 1991 London field penetration in heavy fermion superconductors Z. Phys. B - Condensed Matter 82 243255

[44] Talantsev E F, Iida K, Ohmura T, Matsumoto T, Crump W P, Strickland N M, Wimbush S C and Ikuta H $2019 P$-wave superconductivity in iron-based superconductors Scientific Reports 914245

[45] Hirsch J E and Marsiglio F 2015 Hole superconductivity in $\mathrm{H}_{2} \mathrm{~S}$ and other sulfides under high pressure Physica C $\mathbf{5 1 1}$ 45-49

[46] Souza T X R and Marsiglio F 2016 Systematic study of the superconducting critical temperature in two- and three-dimensional tight-binding models: A possible scenario for superconducting $\mathrm{H}_{3} \mathrm{~S}$ Phys. Rev. B 94184509

[47] Harshman D R and Fiory A T 2017 Compressed $\mathrm{H}_{3}$ S: inter-sublattice Coulomb coupling in a high- $T_{\mathrm{c}}$ superconductor J. Phys.: Condens. Matter 29445702

[48] Kaplan D and Imry Y 2018 High-temperature superconductivity using a model of hydrogen bonds Proc. Nat. Acad. Sci. 115 5709-5713 